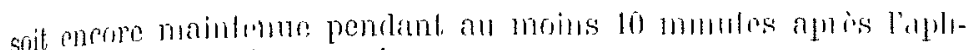
gation to la tension mavina.

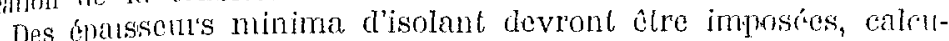
jpes de telle façon que l'isolant ne travalle dans aucune de ses parties à plus de 750) volts par millmètro lors de l'essai de duréc, partions de jo000 volts sous l'essai de falskon maxima re ducé

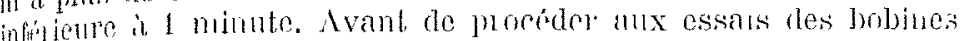

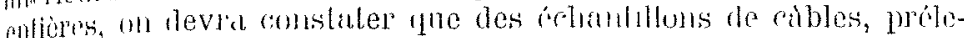
vis sur ces bobines, ne doment pas lien is ruphue avec mo lonsion sunerreure d'nu moins 50 pom 100 a la fension maxima presspite, celle lension étant appliquée graduellement eu partant we lo teluson maxima preserite of l'augmenlant, de 10 pour loo chapur dem-mimule jusru'il ce que la rupture se produise.

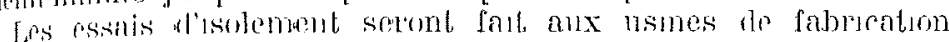

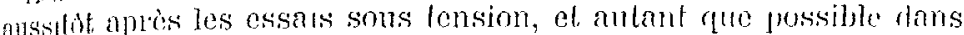

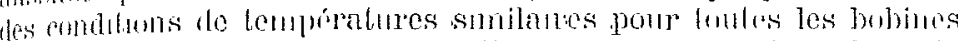

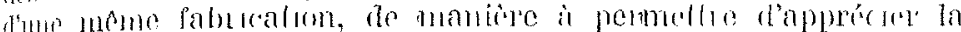

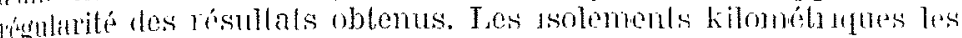

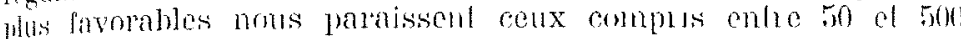

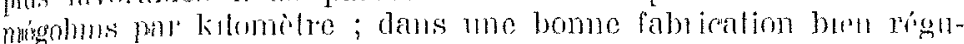

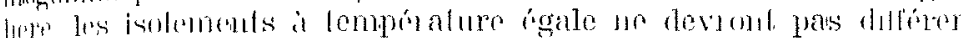
de plut de 20 it 25 pour 100 pour les cables it 50 mégohms, wi do

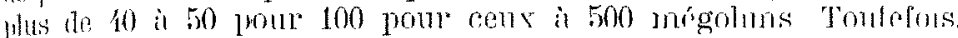

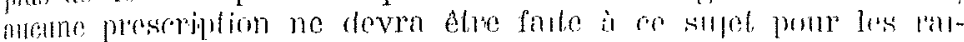
son' dent hous avoms dija exprosios.

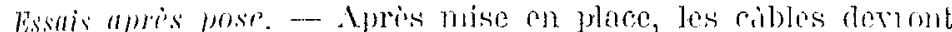

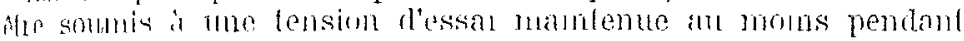
thene an dubble de la lension de servire. Il ne sera pas manras. pour aulant rue les circonslances le permettont, de ne pro-

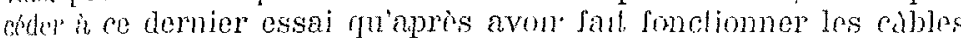

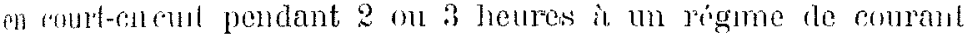

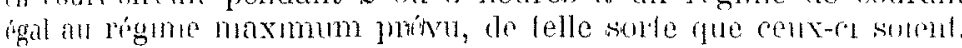

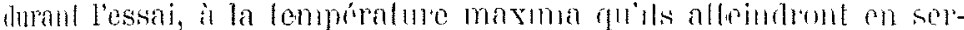
vire courant.

Les diffoules lonsions d'essuis anx usines, of anries pose, indi-

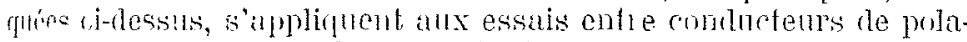
hlis nifferenter des cubles it conduclenes multiples. Dans les ins-

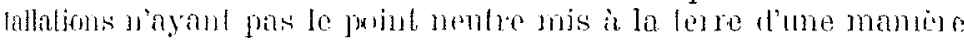

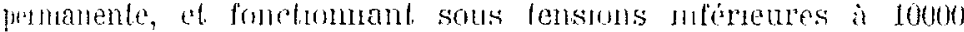

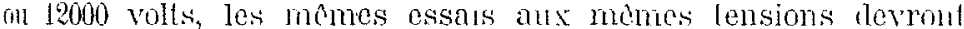
the effectités entre conductem et enveloppe en plomb.

pon les voltages supérieurs d 12000 volls, el mfémeums a 20000 volls, ces lonsions pouront che ziduiles de 10 pour 100

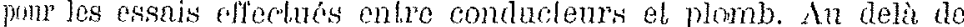
2000 volls, celle rixluction pourra dite pollec à 20 pour 100 . Cos liductoms sonl juslifiables anx tris hantes lensions, parce ofue la capaciti du réseau prend alors me telle valem que le point nonlic se lrumve funjours a pen près an potentiel de la terre. el

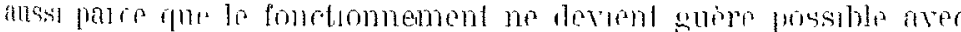
thn defant lisolement sul' une phase.

boilleurs, romme nous liavons dit. aux dis hates tensions,

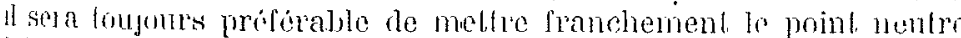

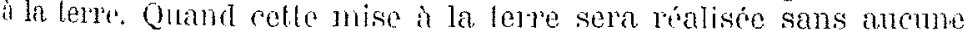
interealation re misistance, les tensions prescrites pourronl atro riflules de 30 poul 100 potir les essais entre conducterurs of

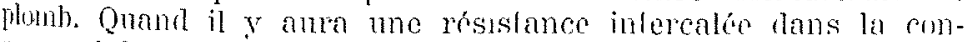
nevion is la terre, ja riduction possible pourra varicr to 20 a 30 poup 100 sinvant l'mmorlane de celle vísistance.

f'ables Tramished rambric et an caoulchour. - Tos cibles Varnished cambric offrent, par leur fabrication mome, plus do gantre de rogulamtí que les cables an papier, of ils sont moins siljels a se détíriorer durnont la mise en place: on oulre, ils ont

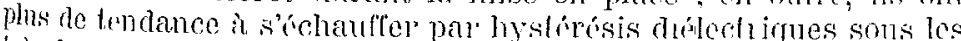
his hantes tensions. Pour ces deux raisons il sora preforable de

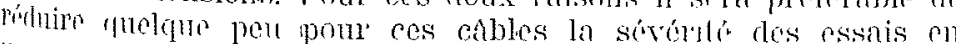
usmes. Par exemple, la formule : $\mathrm{V}=1$ (jomo $+2 \mathrm{~V}$, pouma nnoir son terme constanl réduil, et atro remplacio par la formule $V=5000+2$ E. 1 part celte mortification, fonfes les alutues rondilions d'essai déju indiquées pour les cibles au papier pourlont ctre conscrvées sans changement.

Pour des motifs similaires, les tensions dessais des ribles on choutchouc pourront ctre un peu moindres que celles consellices poin les câbles an papier, surtout quand los essais seronl proscrits apres immersion dans l'ent : ces tensions pourumt alro los mamps que colles indiquées pour les rables rambric.

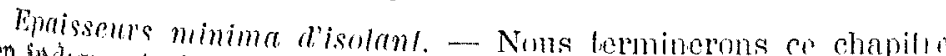
en momant ci-apres les f́passeurs minime d'solants entre doms

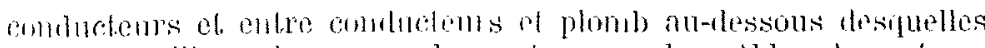

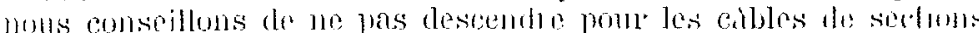
complises calre 30 at $150 \mathrm{~mm}$. par combudeurs, devant fonctumner it tensions de 5000 volts et all-dessus, el elre summis aux essais prescrits ci-clessus. Cess molications sont valables aussi ben

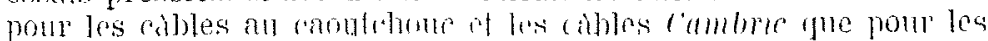
cibles an papiop.

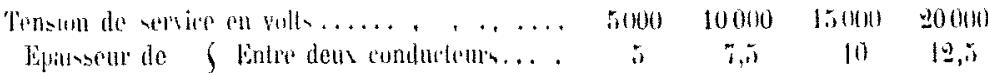

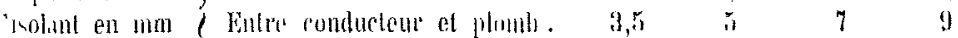

1 shinerel

\section{EXPOSITION DE MARSEILLE}

(Suite)

\section{MINISTÉRE DE L'AGRICULTURE}

La Direction de l'ilydraulique et des Améliorations agricoles avait ol'ganisé deux sections très intéressantes. La première de ces sections était consacrée au Sorvice d'Elude des grandes forces hydrauliques, la seconde au service des Amoliorations agricoles.

L'exposition du service d'Ltude des grandes forces hydrauliques, organiséc par $M$. de La Brosse, ingénieur

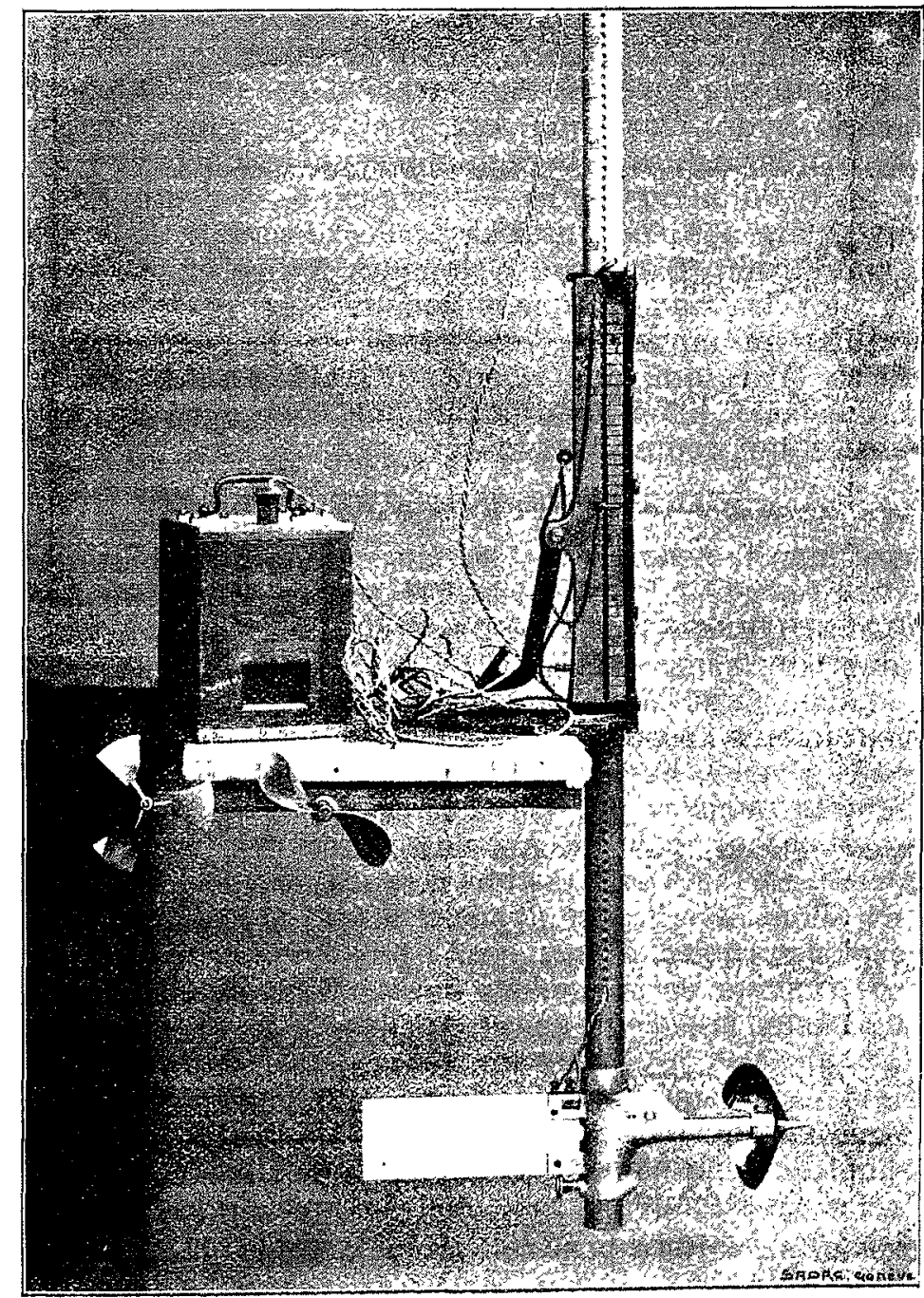

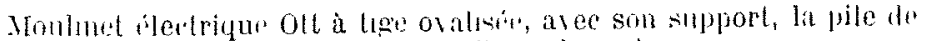
le sonnerje et sos liélies de rechangr.

en chef des Pouts el Chaussies, chef de ce service d'étude pour la rúgion des Alpes, était des plus remarquables, ct comportail 58 pieces, réparlies en 4 groupes.

10 Le groupe des modeles comprenait un relief au $1 / 100$ de la station do jaugeage du pont de la Beaume, sur la Durance, a sisteron; un relief au $1 / 500$ de 19 station do 
jaingeage de Ponsonnas, sur lo Drac; une réduction au $1 / 20$ des barques employées pour les jaugeages; ot uno léduction au $1 / 10$ du manègo de tarage des moulinets, installé sur le fossé d'enceinte do la villo de Greuoble, à la porte Mallifaud.

$2^{\circ}$ Le groupe des appareils el instruments comprenait un limnigraphe ot un hydrometre, tous deux enregistrours, de la maison Richard, des cichelles d'itiage; des moulinets de IVolmann, de Richard, de O tl; un tube jaugeur PitotDarcy, arec échelle graduée en vitesse, du type de $M$. de La Brosse; un hydrotachymètre Ritter; ot divers arcessoirus.

3o Le groupe des Cartes, pholographies et arsinis, comprenait une carte au 1/300000 des usines hydrauliques de la région des Alpes; une carte au 1/500000 des stations de jaugeages de cette même région, et une carte au 1/500000 des usines hydranliques de la l'égion pyrénéenne. Co groupe comprenat aussi dos profils on long, graphiques de régime, courbes de débit, courbes de larages, et photographies diverses.

4" Le groupe des brochures el publications comprenait 22 ouvragres imprimés, parmi lesquels nous citerons les comples rendus duService d'itude des grandes forces hydrauliques de la région des Alpes, pal MM. de La Brosse et Tavernier ( 1905 , et l'Etat statistique des irrigations et des usines existant sur les cours d'eau de la légion pyrénéenne, par $\mathrm{M}$. De Thélin (1908).

I.'Exposition du Service dos Amélioralions agricoles ne comprenait que 9 pièces: Photographie des applications de l'énerrie hydro-électrique aux usagres agicoles; Carte et notice sur les usines de la région normande; Carte et notice sur les installations hydro-électriques de la commune de Neuve-Maison (Aisne), sur loise, et de la Cerme du Courtillet, a Saint-Firmin (Oise), sur la Nonette; Notice et photographies des travaux d'alimentation en esu potable, avec élévation au moyen de l'électricité, de la commune de Cadière (Var) qui, auparavant, n'avait d'autre eau, en été, que celle qu'on apportait de fort loin avec des tonneaux, et qui coûtait très cher.

\section{CANALISATIONS ÉLECTRIQUES}

Les importantes maisons de fabrication de cables électriques: Société industrielle des Téléphones (Paris), Geoffroy et Delore, (Paris), Berthoud-Bolel et Cie (Lyon), Ia Canalisation Electrique (Anciens Etablissements $G$ et II - B. de la Mathe, Saint-Maurice (Seino), Société Alsacienne de Constructions mécaniques (Belfort), Etablıssements AubertGrenier (Paris), exposalent divel's ècliantillons de leurs différents modèles de câbles et de fls électriques. Nous ne donnerons pas la nomenclature des echantillons exposés, car ce serait fastidieux pour nos lecteur's, mais nous allons décrire un appareil assez origiral, destiné à lessai des câhles à très haute tension, qui fonctionnait dans le stand de la maison Berthoud-Bolel, et qui est dî a M. DEıoN, ingénieur do cotte maison.

Cet appareil est hasti sur le principe suivant: Deux points $A$ el $B$ sont soumis à une différence de potentiel alternalive, de valeur efficace $t '$. Si l'un des points, A par oxemple, est maintenu a un potenticl fixe, cn le meltant à la terre par exemple, le potentiel du point $B$, par rapport au point A, variera pendant chaque période, de $+H^{\prime} V \overline{2} \dot{a}$ - $E V$ 2. Si maintenant, au mojon dun contact instantané, l'on charge l'une des armatures d'un condensatcur lorsque la tension estégale $\dot{a}+i V$, et l'autre armature lorsque la tension est égale à $\quad i^{\prime} V \bar{T}$, la différenco de potentiel entro les deux armatures atteindra la valeur 2 I $V \overline{2}$, et ce maintiendra fixe si les déperditions d'électricile a travers le diejlertrique sont négligeables devant la cquantité d'électricité qui vient affuer sur charue armatuse à chaque nouvelle charge. leb appareil est comme on le voil fort simple. Il - e com. pose essentiellement d'un cylindro on ćbonite qui tourne a la moitié de la vites $i c$ de pulsation du courant, el foni porte des bras inétalliques permettant d'envoyer au momeni voulu le courant surchacune des armatures du conden. sateur, ces armatures étant ici constituées par les conduc. teurs d'un câble figurant un câble en essai. Ces bras viennent on contact avec 4 bolnos fixéos sur un relcle également en ébonite. boux de ces bornes, à 90" l'une de l'autre, sont reliées à l'uu des pôles d'un transformateur, qui constitue lo point B précité; l'autre pôle, consitituant le pont $\mathrm{A}$, est a la terre. Les deux autres hornes, diamótrile. mont opposées aux précodontes, sontreliéos chacune a l'un des conducteurs du cible à issayer. L.es bras mélal. liques étant radiaux, et dans le prolongement l'un de l'autre, itablissent deux fois par tour le contact entre les bornes diamétrales

Lo cylindre est entrainé par un moteur synchrơne, aljmenté par la méme source do courant quo le transformateur, de sorte que le réglage une fois fart se maintiont indéfinment. le réglage s'effectue par tâtomnoment, en déplacant le cercle qui porte les bornes. Pratiquement, on dispose deux éclateurs à une distance délerminée et, la tension aux bornos primaires du transformateur ayant amenćo à la valcur fixée, on déplace le cercle cn ébonite juscru’à ce culo la décharge so produise.

A Marsenlle, la tension etait ćlevie de 190 volts a 110000 volts par le transformateur statique, de sorte qu lion réalisait, entre les conducteur's du câble à cscaycr, une différence de potentiel d'un peu plus de 300000 volts, ce cul permettait d'obtenir entre les éclateurs des ćtincollos do trente centimètros. Ces étincelles, en éclatant,provorfuatent un bruit assourdissant qui s'entendait,non seulement d'un bout à l'autre du Palais de l'Enorgie, mais même de lextérieur.

M. P.

\section{SUR LA COMPARAISON DES DIFFÉRENTS MODES DE TRANSPORT DE L'ÉNERGIE ÉLECTRIQUE}

Communcation faite au Congres t'Electriciti do Marselle

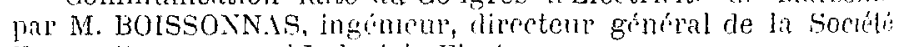

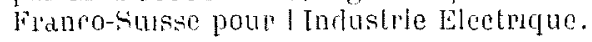

Ia suporiorilo des rourants liphlases pour le transport do l'eble

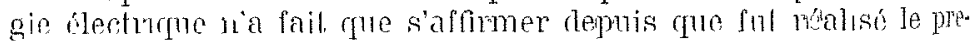
nure lranspot, 1 y a ì peine un quarl de slècle. Il est mome snp-

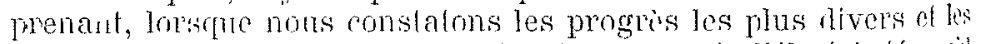

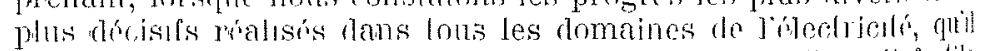

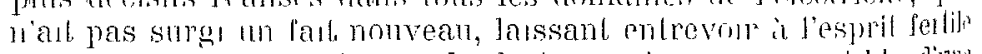

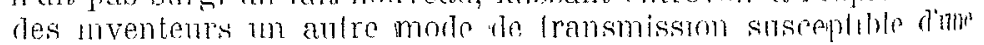
application aussis gincirale.

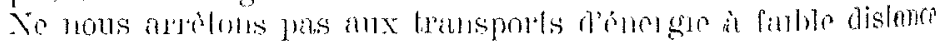
ot a leurs applicalions à l'éclarage.

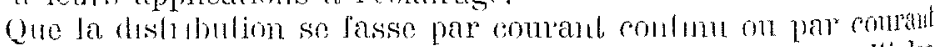

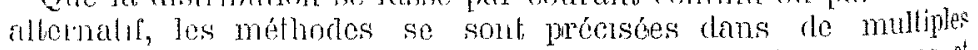
"ximples qui ont tonne des rigles manimemont poommes is

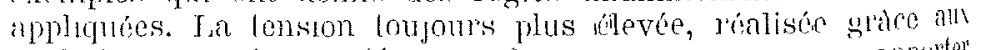

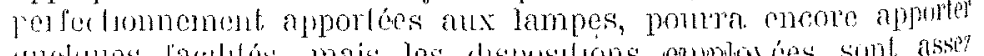

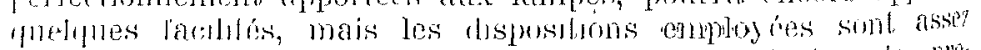
parfales pour résoutle dine manere salisfaisante lous les pro bines qu se josent dans les dishbihulions les plus romplexes

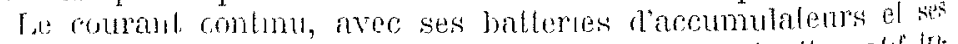

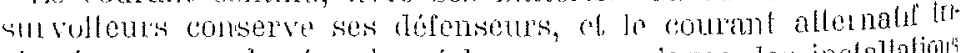
jhasó on monophassó a donno la prenve qu'aver dos inslathation julsisules an service parfal peul. bhe realsst.

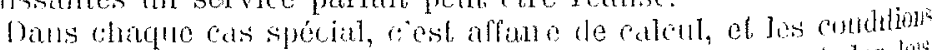

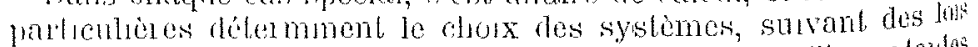

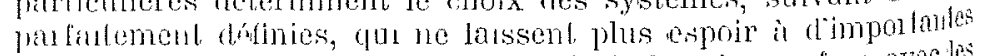

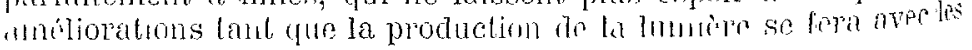
riethodes acturloment employés. 